\title{
Enhanced spin Hall effect by resonant skew scattering in orbital-selective Kondo effect
}

\author{
Guang-Yu Guo, ${ }^{1}$ Sadamichi Maekawa, ${ }^{2,3}$ and Naoto Nagaosa, ${ }^{4,5, \text {, }}$ \\ ${ }^{1}$ Department of Physics, National Taiwan University, Taipei 106, Taiwan \\ ${ }^{2}$ Institute for Materials Research, Tohoku University, Sendai 980-857r, Japan \\ ${ }^{3}$ CREST, Japan Science and Technology Agency, Tokyo 100-0075, Japan \\ ${ }^{4}$ Department of Applied Physics, The University of Tokyo, Tokyo 113-8656, Japan \\ ${ }^{5}$ Cross-Correlated Materials Research Group (CMRG), ASI, RIKEN, Wako 351-0198, Japan
}

\begin{abstract}
The enhanced spin Hall effect in Au metal due to the resonant skew scattering is studied with first-principles band structure calculations. Especially the gigantic spin Hall angle $\gamma_{S} \cong 0.1$ observed recently (T.Seki et al., Nature Materials 7, 125 (2008)) is attributed to the orbital-dependent Kondo effect of $\mathrm{Fe}$ in the Au host metal, where the $t_{2 g}$-orbitals are in the mixed-valence region while $e_{g^{-}}$ orbitals are in the Kondo limit. The enhanced spin-orbit interaction by the electron correlation in the $t_{2 g}$-orbitals leads to the gigantic spin Hall effect. Impurities with $5 \mathrm{~d}$ orbitals are also discussed.
\end{abstract}

PACS numbers: 71.70.Ej, 75.30.Kz, 75.80.+q, 77.80.-e

Spin Hall effect (SHE) is an effect where the transverse spin current is produced by the electric field or electric current [1, 2, 3, 4, 15, 6, 7, ㅇ, 9, 10, 11, 12, 13, 14, 15]. It does not require any magnetic field or magnets, and is an interesting and promising phenomenon for application to spin injection and manipulation in spintronics. SHE is especially robust and large in metals due to the large number of carriers and Fermi energy 10, 11, 12, 13, 14, 15]. For example, SHE in Pt metal even at room temperature [12, 14 is more than 2 orders of magnitude larger than that of GaAs [7]. Even larger SHE has been recently reported in $\mathrm{Au} / \mathrm{FePt}$ System [13], where the spin Hall conductivity is $\sim 10^{5} \Omega^{-1} \mathrm{~cm}^{-1}$ and the spin Hall angle $\gamma_{S}$ is as large as $\cong 0.1$.

Naively speaking, SHE is the two copies of anomalous Hall effect (AHE) for up and down spins, respectively, and the knowledge on the latter can be directly transferred to the former. When the longitudinal conductivity $\sigma_{x x}$ is larger than $\sim 10^{6} \Omega^{-1} \mathrm{~cm}^{-1}$, and the Hall conductivity $\sigma_{H}$ is much larger than $e^{2} /(h a) \cong 10^{3} \Omega^{-1} \mathrm{~cm}^{-1}$ with $h$ and $a$ being the Planck constant and the lattice constant, respectively, the dominant contribution to the AHE is the extrinsic skew scattering [16, 17, 18, 19, 20]. In this case, $\sigma_{H}$ is proportional to $\sigma_{x x}$, and hence the Hall angle $\gamma=\sigma_{H} / \sigma_{x x}$ is the well-defined measure for the magnitude of AHE independent of the impurity concentration. In other words, the Hall angle can be determined by examining the single scattering event due to the impurity. The typical value of $\gamma$ is estimated as the ratio $\sim \lambda / \varepsilon_{F}$ with $\lambda$ being the spin-orbit interaction (SOI) and $\varepsilon_{F}$ the Fermi energy. Usually, $\varepsilon_{F}$ is at least a few $\mathrm{eV}$, the $\lambda$ is of the order of $10-20 \mathrm{meV}$ for the $3 d$ orbitals, for example. Therefore, the Hall angle is roughly estimated as $\gamma \sim 10^{-3}$. This Hall angle $\gamma$ can be enhanced by the resonant skew scattering by the magnetic impurity [17, 18]. Using the Anderson Hamiltonian describing the virtual bound state causing the resonant scattering, the anomalous Hall angle can be expressed in terms of the hybridization energy $\Delta$ and SOI $\lambda$, and the phase shift $\delta_{1}$

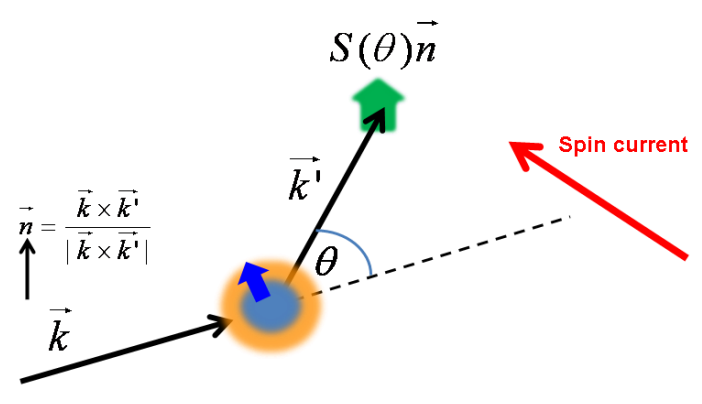

FIG. 1: (color) The skew scattering due to the spin-orbit interaction of the scatterer and the spin unpolarized electron beam with wavevector $\vec{k}$ with the angle $\theta$ with the spin polarization $S(\theta) \vec{n}$ with $\vec{n}=\left(\vec{k} \times \vec{k}^{\prime}\right) /\left|\vec{k} \times \vec{k}^{\prime}\right|$.

due to the $p$-wave scattering as $\gamma \sim(\lambda / \Delta) \sin \delta_{1}$, which can be of the order of $10^{-2}$ [17]. Therefore, the spin Hall angle $\gamma_{S} \cong 0.1$ is a surprisingly large value, which needs to be understood for the design of the gigantic SHE.

In this paper, we propose that the local electron correlation and spin fluctuation further enhance SHE compared with AHE by the explicit first-principles band structure calculation with the Au metal as the host where the Fermi energy is at the $6 s$ bands. Namely, SHE is not the simple two copies of AHE, and the comparison between these two offers a unique opportunity to study the many-body effect.

Let us consider the scattering of a spin $S=1 / 2$ particle by a potential with SOI. The amplitude of the scattered wave is given by 16

$$
\begin{aligned}
& f_{\uparrow}(\theta)=f_{1}(\theta)|\uparrow\rangle+i e^{i \varphi} f_{2}(\theta)|\downarrow\rangle \\
& f_{\downarrow}(\theta)=f_{1}(\theta)|\downarrow\rangle-i e^{-i \varphi} f_{2}(\theta)|\uparrow\rangle
\end{aligned}
$$

for incoming up-spin and down-spin electrons, respectively, where $\theta$ is the angle between the wavevectors of incident $(\vec{k})$ and scattered $\left(\vec{k}^{\prime}\right)$ waves, and $\left.f_{1}(\theta)\right)\left(f_{2}(\theta)\right)$ corresponds to the spin non-flip (spin flip) scattering amplitudes. The skewness of the scattering is represented 
by the function

$$
S(\theta)=\frac{2 \operatorname{Im}\left[f_{1}^{*}(\theta) f_{2}(\theta)\right]}{\left|f_{1}(\theta)\right|^{2}+\left|f_{2}(\theta)\right|^{2}},
$$

while the function $I(\theta)=\left|f_{1}(\theta)\right|^{2}+\left.f_{2}(\theta)\right|^{2}$ determines the scattering intensity. The meaning of the skewness function $S(\theta)$ is shown in Fig.1. When the unpolarized electrons are incident, the scattered electrons are spin polarized as $S(\theta) \vec{n}$ with $\vec{n}=\left(\vec{k} \times \vec{k}^{\prime}\right) /\left|\vec{k} \times \vec{k}^{\prime}\right|$. By solving the Boltzmann equation with this skew scattering, the spin Hall angle $\gamma_{S}$ is given by [9]

$$
\gamma_{S}=\frac{\int d \Omega I(\theta) S(\theta) \sin \theta}{\int d \Omega I(\theta)(1-\cos \theta)}
$$

where $\int d \Omega$ is the integral over the solid angle. The numerator in eq.(3) represents the transverse spin current produced by the scattering, i.e., the velocity perpendicular both to $\vec{k}$ and $\vec{n}$ with the spin polarized along $\vec{n}$, while the denominator corresponds to the usual transport scattering rate. Without the resonance effect, the typical value of $\gamma$ is of the order of $10^{-3}$, i.e., much smaller than unity. Actually, the obtained value of $\gamma$ in ref. [9] is 1/500 for n-type GaAs assuming the screened Coulomb potential. The partial wave analysis gives the expression for $f_{1}(\theta)$ and $f_{2}(\theta)$

$$
\begin{aligned}
& f_{1}(\theta)=\sum_{l} \frac{P_{l}(\cos \theta)}{2 i k}\left[(l+1)\left(e^{2 i \delta_{l}^{+}}-1\right)+l\left(e^{-2 i \delta_{l}^{-}}-1\right)\right] \\
& f_{2}(\theta)=\sum_{l} \frac{\sin \theta}{2 i k}\left(e^{2 i \delta_{l}^{+}}-e^{2 i \delta_{l}^{-}}\right) \frac{d}{d \cos \theta} P_{l}(\cos \theta)
\end{aligned}
$$

Putting eq.(4) into eq.(3), we obtain

$$
\gamma_{S}=\frac{3}{2} \frac{\operatorname{Im}\left[\left(e^{-2 i \delta_{1}}-1\right)\left(e^{2 i \delta_{2}^{+}}-e^{2 i \delta_{2}^{-}}\right)\right]}{9 \sin ^{2} \delta_{2}^{+}+4 \sin ^{2} \delta_{2}^{-}+3\left[1-\cos 2\left(\delta_{2}^{+}-\delta_{2}^{-}\right)\right]}
$$

where we have assumed that the resonant channel is the $d$-wave $(l=2)$ which is subject to SOI and the scattering is characterized by the two phase shifts $\delta_{2}^{ \pm}=\delta_{J=2 \pm 1 / 2}$, while that $\left(\delta_{1}\right)$ for the $p$-wave scattering is assumed to be spin-independent. Assuming that $\delta_{1}$ for the nonresonant $p$-wave scattering is small $\left(\left|\delta_{1}\right| \cong 0.1\right.$ [17]), $\gamma_{S} \cong-3 \delta_{1}\left(\cos 2 \delta_{2}^{+}-\cos 2 \delta_{2}^{-}\right) /\left(9 \sin ^{2} \delta_{2}^{+}+4 \sin ^{2} \delta_{2}^{-}+\right.$ $\left.3\left[1-\cos 2\left(\delta_{2}^{+}-\delta_{2}^{-}\right)\right]\right)$. Therefore, the most important factor is $\cos 2 \delta_{2}^{+}-\cos 2 \delta_{2}^{-}$, which is related to the difference in the occupation numbers of the $J=2 \pm 1 / 2$ impurity states induced by SOI through the Friedel sum rule 21]. Therefore, the local density of states (DOS) for the $d$-electrons determines the magnitude of SHE, which can be studied by the first-principles band structure calculation as described below.

We take $\mathrm{Au}$ as the host metal, where the gigantic SHE has been observed [13]. The electron configuration of $\mathrm{Au}$
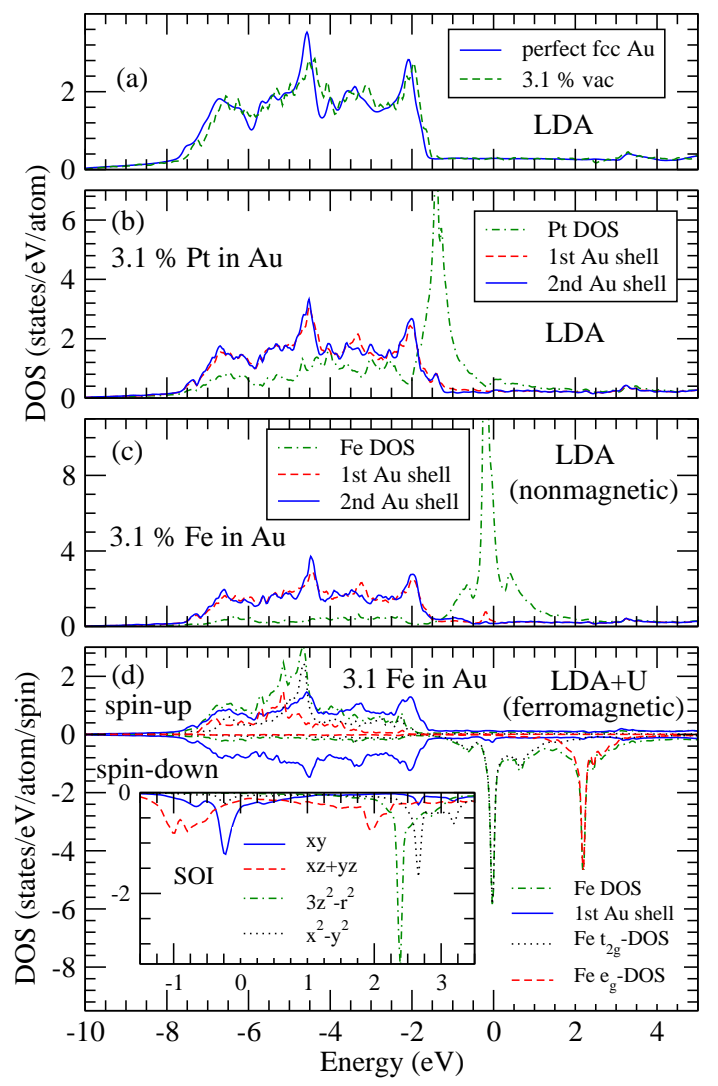

FIG. 2: (color) Total, site-, orbital- and spin-decomposed DOSs of (a) bulk fcc Au, $3.1 \%$ Au-vacancies, (b) $3.1 \%$ Ptimpurities, (c) $3.1 \%$ Fe-impurities in nonmagnetic state and (d) in ferromagnetic state.

atom is $5 d^{10} 6 s^{1}$, and the Fermi energy is at the $6 s 6 p$ bands with relatively small SOI. Therefore, it is difficult to explain the giant SHE in terms of the intrinsic mechanism, in sharp contrast to the case of Pt with $5 d^{9} 6 s^{2}$ [14]. Because in [13] the spin current is supplied from $\mathrm{FePt}$ to $\mathrm{Au}$, it is natural to consider the three possibilities of the imperfections, i.e., (i) $\mathrm{Au}$ vacancies, (ii) $\mathrm{Pt}$ impurities, and (iii) Fe impurities. From the above consideration, there are several requirements to obtain the gigantic SHE; (I) there should be the resonance at the Fermi energy due to either the Kondo peak or the mixed valance, (II) the orbital angular momentum should not be quenched, and (III) the peak must be split due to SOI by the energy comparable or larger than the width of the peak. We have studied the impurity states in Au host metal for these three cases in terms of the local density approximation (LDA) 27] plus on-site Coulomb interaction $U(\mathrm{LDA}+U)$ [28] $(U=5, J=0.9 \mathrm{eV})$. We used the highly accurate full-potential augmented plane wave method, as implemented in the WIEN2k code 29]. The atomic positions are relaxed in the presence of the impurity. The numerical results presented below have been tested for convergence with respect to the energy cut-off for augmented plane waves, $k$-points used for irreducible 
TABLE I: Down-spin occupation numbers of the $3 d$-orbitals of the $\mathrm{Fe}$ impurity in $\mathrm{Au}$ from $\mathrm{LDA}+U$ calculations without SOI and with SOI. The calculated magnetic moments are: $m_{s}^{F e}=3.39 \mu_{B}$ and $m_{s}^{\text {tot }}=3.32 \mu_{B}$ without SOI, as well as $m_{s}^{F e}=3.19 \mu_{B}, m_{o}^{F e}=1.54 \mu_{B}$ and $m_{s}^{\text {tot }}=3.27 \mu_{B}$ with SOI. The muffin-tin sphere radius $R_{m t}=2.65 a_{0}$ ( $a_{0}$ is Bohr radius) is used for both $\mathrm{Fe}$ and $\mathrm{Au}$ atoms.

\begin{tabular}{cccccc}
\hline \hline (a) & $x y$ & $x z$ & $y z$ & $3 z^{2}-r^{2}$ & $x^{2}-y^{2}$ \\
no SOI & 0.459 & 0.459 & 0.459 & 0.053 & 0.053 \\
SOI & 0.559 & 0.453 & 0.453 & 0.050 & 0.128 \\
\hline (b) & $m=-2$ & $m=-1$ & $m=0$ & $m=1$ & $m=2$ \\
no SOI & 0.256 & 0.459 & 0.053 & 0.459 & 0.256 \\
SOI & 0.138 & 0.087 & 0.050 & 0.819 & 0.549 \\
\hline \hline
\end{tabular}

Brillouin zone and supercell size. Figure 2 shows the density of states (DOS) in the presence of one impurity in the $2 \times 2 \times 2$ supercell. Basically the structure extending from $-8 \mathrm{eV}$ to $-2 \mathrm{eV}$ is due to the $5 d$ bands of $\mathrm{Au}$, while $6 s 6 p$ bands of $\mathrm{Au}$ are extended all through in this energy range with smaller DOS. The change in the DOS is almost confined in the range of the $5 d$ bands in the case of (i) vacancy (Fig.2(a)), and is at around $-1.5 \mathrm{eV}$ in the case of (ii) Pt (Fig.2(b)). We could not obtain a magnetic state in both of these cases even in the $\mathrm{LDA}+U$ calculation. This conclusion is consistent with the earlier study by photoelectron spectroscopy [22]. In sharp contrast, we obtained the magnetic state as the ground state with both the LDA and LDA $+U$ calculations in the case of Fe. Accordingly, the DOS for Fe (Fig.2(d)) shows the spin splitting, and the down spin DOS has the sharp peak close to the Fermi energy. (We put the DOS for nonmagnetic state in Fig.2(c) for reference.) This means that there occurs the valence fluctuation of Fe ion between $d^{6}$ and $d^{7}$. This is reasonable since the $\mathrm{Fe}$ in $\mathrm{Au}$ is known as a Kondo impurity but the Kondo temperature is as low as $0.4 \mathrm{~K}$. From these results, we conclude that only (iii) Fe impurities could the origin of the large SHE satisfying the criteria (I),(II),(III) discussed above, and we now analyze Fe impurity in Au in more detail below.

The band structure calculation for the state with local magnetic and orbital orderings corresponds to the mean field theory of the Anderson Hamiltonian 21]. In the limit of isolated impurity atom, this mean field theory gives the correct energy positions of the peaks in the spectral function of the single-particle Green's function. Of course, there is no symmetry breaking by the local electron correlation, and the true ground state is the quantum mechanical superposition of the degenerate symmetry breaking states, i.e., quantum fluctuation of spins and orbitals occur. Early theories of Kondo effect of $\mathrm{Fe}$ in $\mathrm{Au}$ have estimated the relevant quantities as $\Delta \cong 1.4 \mathrm{eV}, U \cong 5.4 \mathrm{eV}, J \cong 0.9 \mathrm{eV}$ 23], where $\Delta$ is the energy broadening of the virtual bound state due to the hybridization with the conduction bands, $U$ is the on-site Coulomb interaction, and $J$ is the Hund's coupling energy between different orbitals. The crystal field splitting is considered to be small $(\sim 0.1 \mathrm{eV})$, and SOI $\lambda \cong 30 \mathrm{meV}$ is even smaller. The resistivity measurement at room temperature shows a systematic change as the valence of the impurity changes as $\mathrm{Ti}, \mathrm{V}, \mathrm{Cr}, \mathrm{Mn}, \mathrm{Fe}$, $\mathrm{Co}, \mathrm{Ni}$, and shows the dip at $\mathrm{Mn}$, while the maximum at Fe (Fig. 17 of Ref. 24). This strongly suggests the peak in the DOS for Fe, i.e., the mixed valence case, although the low temperature properties have been analyzed by the Kondo model 24]. The magnetic susceptibility measurement shows the $S=2$ magnetic moment, while the Moessbauer experiment concluded rather $S=3 / 2$ [25]. These somewhat confusing situation can be resolved by the LDOS in Fig. 2(c-d) together with Table I. Table I shows the spin/orbital magnetic moments and the occupation number of each $d$-orbital in terms of LDA + $\mathrm{SOI}+U$ calculation. Note that the obtained values depend slightly on the muffin-tin sphere radius $R_{m t}$ and $U$ value, but the semi-quantitative conclusion does not change. First, the nonmagnetic state in the LDA calculation shows almost no crystal field splitting (of the order of $0.1 \mathrm{eV}$ ), which is consistent with the earlier result. However, the inclusion of $U$ changes the situation dramatically, and the $e_{g}-t_{2 g}$ splitting is enhanced to be around $2 \mathrm{eV}$ as shown in Fig. 2(d). This corresponds to the orbital polarization due to the electron correlation, i.e., the local version of the orbital ordering not by the crystal field but by the electron correlation [26]. Therefore we conclude that the orbital-dependent Kondo effect occurs for $\mathrm{Fe}$ in $\mathrm{Au}$; the $e_{g}$ orbitals are in the Kondo limit, while $t_{2 g}$-orbitals are in the mixed valence region. Therefore, at temperatures above $T_{K} \cong 0.4 \mathrm{~K}$, the $t_{2 g}$-orbitals, within which the orbital angular momentum is not quenched, play the major role in the transport properties, while the $e_{g}$-orbitals determine the low temperature Kondo effect.

Now we consider the orbital polarization within the $t_{2 g}$ states due to the SOI. Note that $t_{2 g}$-orbitals behaves like $l_{\text {eff. }}=1$ states with $x y, z x+i y z$, and $z x-i y z$ orbitals corresponding to $m=0, m=1$, and $m=-1$ states, respectively. Therefore, SOI is effective within the $t_{2 g}$ states leading to the energy splitting between the $J_{\text {eff. }}=3 / 2$ and $J_{\text {eff. }}=1 / 2$ states. Naively, the orbital polarization is determined by the competition between the hybridization energy $\Delta$ and the energy splitting due to SOI. Here, one must carefully distinguish between the many-body states and the single-particle states. The energy separation between the many-body state with different total angular momentum $J$ is typically the order of SOI, and much smaller than $U$ or $J$. However, once the many-body ground state of $d$-electrons is fixed, the separation of the single-particle state energy, which is the energy difference between the $N$-electron and $N \pm 1$-electron state, can be as large as $U$ or $J$, which is important for the conduction electrons which comes in or out to the $d$-orbitals. Therefore, it is possible that the electron correlation $U$ plays an 
essential role, and even a SOI much smaller than the hybridization energy can produce the large orbital magnetic moment $m_{o}=1.54 \mu_{B}$ as shown in Table I. Correspondingly, the single-particle $m=1$ state is almost occupied (0.819) while $m=-1$ state is almost empty (0.087), as is seen also from the inset of Fig. 2(d). Figure 2(d) and Table I also explain why the AHE is rather small compared with SHE. As for AHE, the phase shift for $m=1$ $(m=-1)$ are almost $\pi(0)$, and both of them does contribute a little to the scattering, while $m=0$ state is almost in the unitary limit with $\pi / 2$-phase shift. Therefore, we do not expect the enhanced AHE, leading to the small anomalous Hall angle compared with that for SHE [17]. For the SHE, on the other hand, such a cancelation does not occur. One needs to treat the spin/orbital fluctuation in this case, but a rough estimate for the spin Hall angle can be obtained as follows. For the conduction electrons, the energy difference between $J_{\text {eff }}=3 / 2$ and $J_{\text {eff }}=1 / 2$ is that of $m=1$ and $m=-1$ states in the mean field theory when one considers the Ising type coupling $l_{z} s_{z}$. This energy is larger than the hybridization energy and we expect the difference of the phase shifts between these two channels of the order of $\pi$, giving a large spin Hall angle of the order of $\delta_{1} \cong 0.1$ as observed experimentally [13]. This picture for the Kondo effect in $\mathrm{Fe}$ impurity in $\mathrm{Au}$ is different from the conventional one 23]. Further experimental studies using the spectroscopies such as STS and ARPES are highly desired to clarify the nature of this fundamental problem.

SHE associated with Kondo effect is expected for other ions such as rare-earth impurity [30]. From this viewpoint, we have also studied the case of $5 d$ impurities, i.e., $\mathrm{Ta}, \mathrm{W}, \mathrm{Re}, \mathrm{Os}$, Ir doped into Au. In these cases, the appropriate $U$ value is around $0.5 \mathrm{eV}$, and there occurs no magnetic state. However, $\mathrm{SOI}$ is almost as large as $U$, and hence the large SHE is expected. The results for the DOS and the associated phase shifts are shown in Fig.3 for W (Fig.3(a)) and Os (Fig.3(b)) as the two representative examples. Even without the magnetic moment, SHE is enhanced due to the resonant state at Fermi energy, and the corresponding spin Hall angle is estimated as $\gamma \cong 0.2 \sin 2 \delta_{1}$ where $\delta_{1}$ is the phase shift for the $p$ wave scattering, and we expect $\left|\gamma_{S}\right| \cong 0.04$ in these cases in semi-quantitatively agreement with the early experiment 17].

In conclusion, we have studied the enhanced SHE due to the resonant skew scattering. Compared with AHE, SHE, where the time-reversal symmetry is not broken, gives a unique opportunity to study the electronic states near the impurity without disturbing its magnetic behavior. We have presented the formula for the spin Hall angle in terms of the phase shifts (eq.(5)), and also the first-principles band structure calculation to analyze the mechanism of the gigantic SHE. We have shown that SHE shed a new light on the Kondo effect, which plays the key role to enhance SHE and a new picture for the Fe impu-

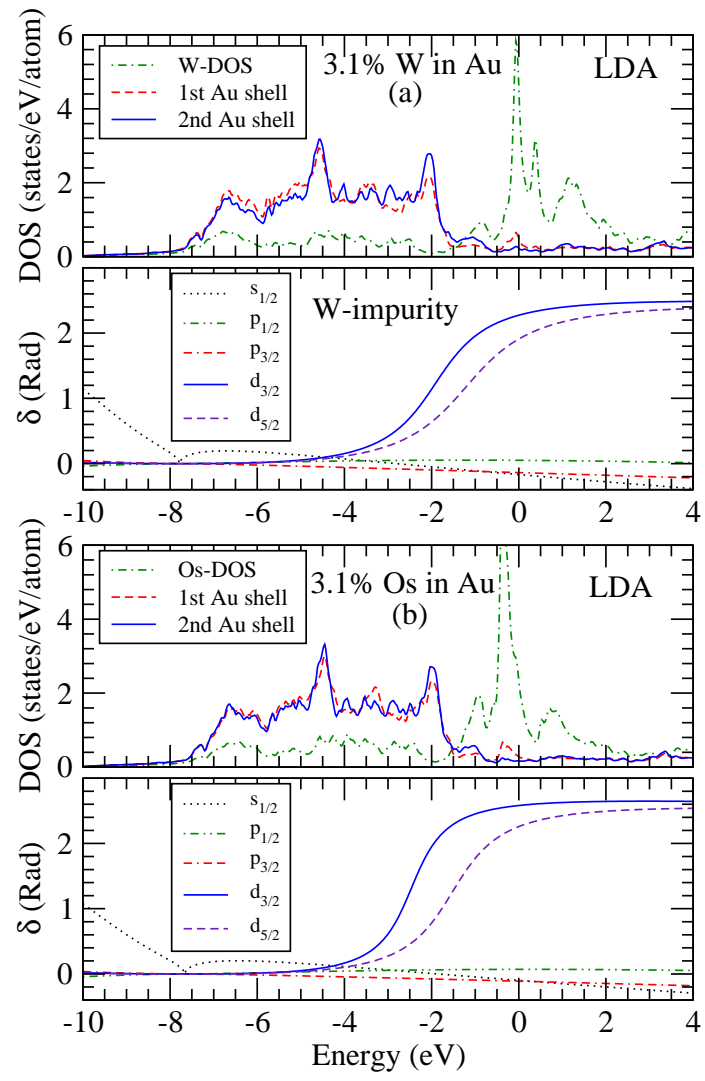

FIG. 3: (color) Site-decomposed DOSs and phase shifts $(\delta)$ of (a) $3.1 \% \mathrm{~W}$ and (b) $3.1 \%$ Os in Au.

rity state in $\mathrm{Au}$ has been proposed. This leads to the material design of the large SHE even at room temperature with the potential application to the spintronics.

We thank Profs. A. Fert, K.Takanashi, N. Kawakami and Y. Otani for fruitful discussions.

* Electronic address: nagaosa@appi.t.u-tokyo.ac.jp

[1] M.I. Dyakonov and V.I. Perel, Sov. Phys. Solid State 13, 3023 (1972).

[2] J. E. Hirsch, Phys. Rev. Lett. 83, 1834 (1999).

[3] S. Zhang, Phys. Rev. Lett. 85, 393 (2000).

[4] S. Takahashi and S. Maekawa, Phys. Rev. Lett. 88, 116601 (2002).

[5] S. Murakami, N. Nagaosa, and S.C. Zhang, Science 301, 1348 (2003).

[6] J. Sinova et al., Phys. Rev. Lett. 92, 126603 (2004).

[7] Y. Kato et al., Science 306, 1910 (2004).

[8] J. Wunderlich et al., Phys. Rev. Lett. 94, 047204 (2005).

[9] H.-A. Engel, B. I. Halperin, and E. I. Rashba, Phys. Rev. Lett. 95, 166605 (2005).

[10] S. O. Valenzuela and M. Tinkham, Nature 442, 176 (2006).

[11] E. Saitoh, M. Ueda, H. Miyajima, and G. Tatara, Appl. Phys. Lett. 88, 182509 (2006).

[12] T. Kimura et al., Phys. Rev. Lett. 98, 156601 (2007). 
[13] T. Seki et al., Nature Materials 7, 125 (2008).

[14] G. Y. Guo, S. Murakami, T.-W. Chen, N. Nagaosa, Phys. Rev. Lett. 100, 096401 (2008).

[15] K. Ando et al., Phys. Rev. Lett. 101, 036601 (2008).

[16] H. A. Bethe and R. Jackiw, Intermediate Quantum Mechanics (The Benjamin/Cummings Publishing Company, Inc. 1986), Chap. 16.

[17] A. Fert and O. Jaoul, Phys. Rev. Lett. 28, 303 (1972); A. Fert, A. Friederich, and A. Hamzic, J. Mag. Mag. Mat. 24, 231 (1981)

[18] P. Coleman, P.W. Anderson, and T. V. Ramarkrishnan, Phys. Rev. Lett. 55, 414 (1985); A. Fert and P.M. Levy, Phys. Rev. B36, 1907 (1987).

[19] S. Onoda, N. Sugimoto, N. Nagaosa, Phys. Rev. Lett. 97, 126602 (2006).

[20] T. Miyasato et al., Phys. Rev. Lett. 99, 086602 (2007).

[21] See for example, A.C. Hewson, The Kondo Problem and Heavy Fermions, (Cambridge University Press, 1993).

[22] D. van der Marel, J. A. Jullianus, and G.A. Sawatsky, Phys. Rev. B32, 6331 (1985)
[23] K. Yoshida, A. Okiji, and S. Chikazumu, Prog. Theor. Phys. 33, 559 (1965).

[24] G. Gruener and A. Zawadowski, Rep. Prog. Phys. 37, 1497 (1974).

[25] T.A. Kitchen and R. D. Taylor, Phys. Rev. B9, 344 (1974).

[26] It is also noted that the orbital-selective Mott transition is now discussed showing that the electron correlation act differently for different orbitals. See A. Koga et al., Phys. Rev. Lett. 92, 216402 (2004) and references therein.

[27] S. H. Vosko, L. Wilk and M. Nusair, Can. J. Phys. 58, 1200 (1980)

[28] A. I. Liechtenstein, V. I. Anisimov and J. Zaanen, Phys. Rev. B 52, R5468 (1995).

[29] P. Blaha, K. Schwarz, G. K. H. Madsen, D. Kvasnicka, and J. Luitz, WIEN2k, An Augmented Plane Wave Local Orbitals Program for Calculating Crystal Properties (Techn. University Wien, Austria, 2002).

[30] T. Tanaka and H. Kontani, arXiv:0808.1459. 